\title{
Sacrosidase Trial in Chronic Nonspecific Diarrhea in Children
}

\author{
Riad M. Rahhal* and Warren P. Bishop \\ Division of Pediatric Gastroenterology, Department of Pediatrics, University of Iowa, Iowa City, Iowa, USA
}

\begin{abstract}
Chronic nonspecific diarrhea in children, or toddler's diarrhea, is a frequently encountered entity in pediatric clinical practice. This disorder remains poorly understood. Suggested etiologies include malabsorption, dietary intake and motility abnormalities. We investigated the use of sacrosidase (a yeast sucrase supplement) in children with toddler's diarrhea. The study outcome was clinical response to sacrosidase supplementation. Children, 1-6 years of age, with toddler's diarrhea were enrolled in an open prospective trial. Stooling patterns were obtained at baseline and while on supplementation. Twelve patients were enrolled out of 40 patients who presented with chronic diarrhea. Eight patients did not respond to standard diet changes and were entered into the trial. With supplementation, 4 out of the 8 patients responded clinically with a decrease in mean daily stool frequency and an improvement in the mean daily stool consistency. Sacrosidase supplementation demonstrated a potential benefit in a subset of children with toddler's diarrhea. Response to sacrosidase may suggest excessive sucrose intake or unrecognized partial or complete sucrase deficiency in some children with toddler's diarrhea.
\end{abstract}

Keywords: Congenital sucrase-isomaltase deficiency, sacrosidase, toddler's diarrhea, chronic nonspecific diarrhea.

\section{INTRODUCTION}

Chronic nonspecific diarrhea in children, or toddler's diarrhea, is frequently encountered in clinical practice. Onset is usually between 6 and 30 months of age with symptom resolution after 5 to 6 years of age. These children typically have at least 2-6 loose daily but maintain good nutritional status except when excessive diet restrictions are imposed [1, $2]$. The diarrhea may cause significant parental anxiety and frustration leading to frequent health care visits [3]. This well recognized disorder remains poorly understood [4]. The diagnosis is often made based on history, lack of abnormal physical exam findings and a limited number of screening tests $[1,2]$. Several factors have been suggested to play roles in the pathogenesis including carbohydrate intolerance, overfeeding, excess fluid and juice intake, low fat intake, intestinal motility abnormalities and increased synthesis or sensitivity to prostaglandins $[2,5]$. Management has traditionally aimed at parental reassurance and changing the child's feeding pattern. Fluid intake is decreased especially that of clear fruit juices presumably because of their sucrose and fructose content. Additionally, fiber and fat intake are usually encouraged $[3,6,7]$. Few studies have suggested that drug therapy, including the use of aspirin, loperamide and mebeverine, may help obtain acceptable defecation patterns [3, 4]. This is rarely employed in clinical practice.

The differential diagnosis of chronic diarrhea in children includes enteric pathogens, postinfectious gastroenteritis, small bowel bacterial overgrowth and malabsorptive disorders. Congenital sucrase-isomaltase deficiency (CSID) is a rare autosomal recessive disease that typically causes chronic

*Address correspondence to this author at the University of Iowa Children's Hospital, Department of Pediatrics, Division of Gastroenterology, 200 Hawkins Dr, 2868 JPP, Iowa City, Iowa 52242, USA; Tel: 319356 2950; Fax: 319353 8967; E-mail: riad-rahhal@uiowa.edu or intermittent watery diarrhea in affected children. It is due to the absence of sucrase-isomaltase enzyme complex on the intestinal luminal surface. The sucrase subunit hydrolyzes the glucose-fructose linkage in sucrose and the $\alpha 1-4$ glucose linkages in maltose and maltotriose. The isomaltase subunit cleaves the $\alpha$ 1-6 glucopyranosyl linkages in branched oligosaccharides, the 1-6 linkages in isomaltase and 1-4 linkages of maltose [8]. Patients with CSID lack sucrase activity but may have variable isomaltase activity $[9,10]$. In addition, the activity of another intestinal disaccharide, maltaseglucoamlyase, overlaps with sucrase-isomaltase in digestion of maltose, maltotriose and starch [11]. These factors most likely play a role in the phenotypic variation among patients with CSID [12].

Prevalence of CSID in the US is $\leq 0.2 \%$ but is higher in certain populations such as Greenland Eskimos (2-10\%) and Canadian native peoples (3.6\%-7.1\%) [13]. It is suggested that this entity is underestimated. Clinical presentation of CSID is variable and in part depends on introduction and content of sucrose in a patient's diet. Treem et al. [13] described 23 cases of CSID in which 7 cases $(30 \%)$ had chronic diarrhea with normal growth. Such children can be misdiagnosed as chronic nonspecific diarrhea, irritable bowel syndrome or cow's milk protein intolerance $[13,14]$. Few reports have documented delayed diagnosis until adulthood [15-17].

The treatment of CSID involves a strict life-long adherence to a sucrose-free diet which is difficult leading to noncompliance and persistent or intermittent symptoms. Starch restriction is not usually needed because of residual isomaltase activity, unless symptoms persist despite implementing a sucrose-free diet $[18,19]$. Sacrosidase, $\beta$ Dfructofuranoside fructohydrolase, is derived from baker's yeast (Saccharomyces cerevisiae) and is a proven oral replacement therapy for patients with CSID $[10,20]$. In its liquid preparation, sacrosidase is extremely potent with very 
high sucrase activity [10]. Such supplementation allows more normal diet consumption in patients with CSID. Excellent symptom relief is usually reached in around $75 \%$ of biopsy proven CSID patients. This high response rate is similar to that noted on a strict sucrose-free diet [20].

A definitive diagnosis of CSID can be made by demonstrating absence of sucrase-isomaltase in the small intestinal biopsy of the distal duodenum or proximal jejunum. Several noninvasive diagnostic methods include the sucrose breath hydrogen test, sucrose tolerance test and differential urinary disaccharidases [13]. Each of these approaches has its advantages and pitfalls. Another possible noninvasive approach to the evaluation of suspected CSID is to conduct a therapeutic challenge with sacrosidase. This is especially helpful in settings where small bowel biopsy with disaccharidase level determination or breath hydrogen testing is difficult or inconvenient to perform.

We hypothesized that a subgroup of patients with chronic nonspecific diarrhea is symptomatic because of a high sucrose intake in their diets or due to unrecognized partial or complete deficiency of intestinal sucrase-isomaltase. We tested our hypothesis by using sacrosidase supplementation as a treatment in patients with chronic nonspecific diarrhea who had not responded to standard diet changes.

\section{METHODS}

\section{Subjects}

Children, 1 to 6 years of age, with toddler's diarrhea were recruited from the outpatient Pediatric Gastroenterology Clinic at the University of Iowa. The study was approved by the institutional review board at our center. Eligibility criteria included toddler's diarrhea defined as having $\geq$ 3 loose stools per day, on at least $50 \%$ of the days over the last 3 months, in the absence of other causes. Exclusion criteria included: (1) symptoms suggestive of infection, inflammation or malnutrition (fever, bloody stools or failure to thrive), (2) known allergy to sacrosidase, yeast, yeast products, or glycerin, or (3) concomitant use of medications that could modify gastrointestinal motility. Use of antibiotics during the four weeks prior to the study period was also an exclusion criterion. Written consent to participate in the study was obtained from parents or legal guardians. All patients had the following screening tests done prior to the intervention: stool culture for enteric pathogens, stool clostridium difficile toxin assay, stool Giardia antigen detection assay, stool Rotavirus antigen assay, complete blood count with differential, C-reactive protein and/or erythrocyte sedimentation rate, tissue transglutaminase IgA, anti-endomysial IgA and a total serum IgA level. Patients with abnormal screening tests were excluded from the study.

\section{Protocol}

Children who satisfied the inclusion criteria for toddler's diarrhea and had normal screening tests were offered enrollment into the study. Parents were provided with daily diaries to fill over one week to assess the following baseline characteristics:

\section{- Stool frequency}

- Stool consistency using a 1 to 5 scale, with $1=$ hard and dry, $2=$ firm, 3 = soft, $4=$ loose, $5=$ watery
- Presence/frequency of abdominal pain, flatulence, nausea or emesis.

A diet free of juices and other sugar-containing beverages and rich in fiber-containing foods was emphasized. Patients whose defecation patterns on this diet satisfied the inclusion criteria were placed on a sacrosidase trial. These patients were prescribed sacrosidase oral solution (Sucraid, QOL Medical, Kirkland, WA, USA) based on weight (1 ml or $8500 \mathrm{IU}$ if $\leq 15 \mathrm{~kg}$; or $2 \mathrm{ml}$ or $17,000 \mathrm{IU}$ if $>15 \mathrm{~kg}$ ) with meals and snacks. The first sacrosidase dose was provided in the clinic and the patients were monitored for 30 minutes for any signs of allergy. Parents were asked to complete daily diaries for 2 weeks while on sacrosidase supplementation. Clinical response was defined by $\geq 50 \%$ decrease in the average number of daily bowel movements and/or an increase (improvement) of $\geq 2$ points on the stool consistency scale. Patients who responded clinically were offered sucrose breath hydrogen testing. An elevation of breath hydrogen by $>20$ parts per million (ppm) over baseline was considered positive after ingesting a sucrose load of $2.0 \mathrm{~g} / \mathrm{kg}$ following an overnight fast.

\section{Statistical Analysis}

Data (age at presentation, duration of symptoms, growth parameters, daily stool frequency and consistency) were expressed as mean values $( \pm \mathrm{SD})$. Other demographic data (gender, ethnicity, presence of family history) were expressed as a percentage of total patients. A two tailed paired $t$ test was used to compare daily stool frequency and consistency before and after the intervention. A value of $P<0.05$ was considered significant.

\section{RESULTS}

Between September 2005 and July 2006, 40 children, 1 to 6 years of age, presented to our clinic with a chief complaint of chronic diarrhea. Of these patients, $12(30 \%)$ satisfied the criteria for toddler's diarrhea by history, physical examination and negative screening tests. Within this cohort, positive family histories were noted for irritable bowel syndrome ( 3 patients), inflammatory bowel disease ( 2 patients) and cystic fibrosis (2 patients). None had a known family history of celiac disease. Demographic data of the patient cohort are presented in Table $\mathbf{1}$.

All families reported their children failed to respond previously to a variety of diet restrictions. After explaining and implementing standard diet changes (elimination of juices and other sugar-containing beverages and increasing fiber intake), diarrhea resolved in three patients as documented by stool diaries. These patients were excluded from further intervention. One family did not return the daily diaries and their child was excluded from the study. Eight patients remained in the study.

The baseline diet characteristics for the remaining eight patients included a mean daily juice intake of 6.8 ounces ( \pm 7.9 ounces, SD), mean daily non-juice fluid intake (such as water, milk) for this group of 15.3 ounces ( \pm 6.7 ounces, SD) and the mean daily carbohydrate food servings (such as bread, pasta, rice, potatoes) of 3.5 servings ( \pm 1.4 servings, $\mathrm{SD})$. These values were not different from subjects who responded to the diet changes. As mentioned previously, parents were instructed to eliminate juice intake completely 
during the study. There were no restrictions placed on water or carbohydrate food intake but increased fiber intake was encouraged. We prescribed sacrosidase to the remaining eight non-responders who completed the 2 week intervention while on the same restricted diet. The first sacrosidase dose was provided in clinic with patients being monitored for 30 minutes. All patients had stable vital signs and none developed any clinical signs of an allergic reaction.

Table 1. Baseline Characteristics of all 12 children with Toddler's Diarrhea

\begin{tabular}{|l|c|}
\hline \multicolumn{1}{|c|}{ Characteristic } & Value \\
\hline \hline Average age, \pm SD (months) & $31.3 \pm 5.7$ \\
\hline $\begin{array}{l}\text { Gender } \\
\text { Males }\end{array}$ & $58 \%$ \\
\hline Ethnicity & $100 \%$ \\
Caucasian & $10.4 \pm 3.7$ \\
\hline Average duration of diarrhea, \pm SD (months) & \\
\hline Growth parameters & $38 \% \pm 27.9$ \\
Weight $\%, \pm$ SD & $39 \% \pm 20.3$ \\
Height $\%, \pm$ SD & \\
\hline Positive family history & $25 \%$ \\
Irritable bowel syndrome & $17 \%$ \\
Inflammatory bowel disease & $17 \%$ \\
Cystic fibrosis & $0 \%$ \\
Celiac disease & \\
\hline
\end{tabular}

A non-significant statistical difference was noted between the mean stool frequency reported by parents during the clinic visit and that obtained by diaries during the baseline period, with $5.4( \pm 3)$ stools per day by history compared to $4( \pm 1.5)$ stools per day by recorded diaries $(P$ value 0.12$)$. The mean daily stool consistency was similar by history and by recorded diaries at $4.3( \pm 0.7)$ and $4.2( \pm 0.8)$, respectively $(P$ value 0.97$)$.

Following the addition of sacrosidase, four out of the eight patients responded clinically with improvement in their stool characteristics (Figs. 1, 2). The mean daily stool frequency for all eight patients decreased from $4( \pm 1.5)$ to $2( \pm$ 0.5 ) ( $P$ value $0.01,95 \%$ CI 0.64 - 3.35). The mean daily stool consistency scale also improved from $4.2( \pm 0.8)$ to 2.9 $( \pm 0.6)(P$ value $0.01,95 \%$ CI $0.40-2.37)$.

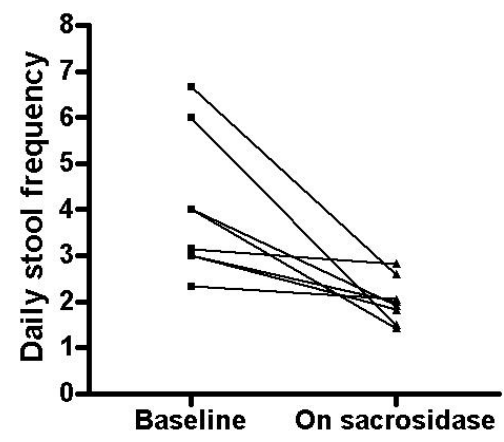

Fig. (1). Stool frequency. The daily stool frequency decreased from a mean of $4( \pm 1.5)$ stools per day at baseline to a mean of $2( \pm$ $0.5)$ stools per day while on sacrosidase supplementation.

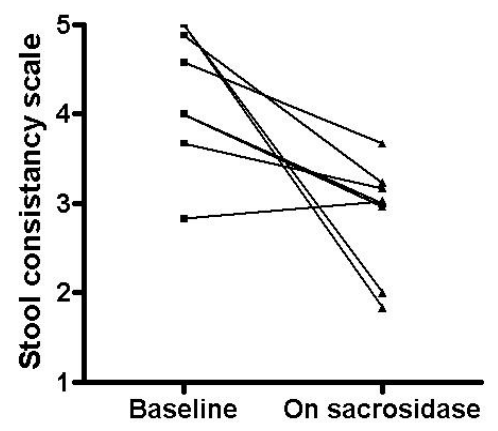

Fig. (2). Stool Consistency. The daily stool consistency score improved from a mean of $4.2( \pm 0.8)$ points (out of a total of 5 points) at baseline to a mean of $2.9( \pm 0.6)$ while on sacrosidase supplementation.

The families of two clinical responders agreed to sucrose breath hydrogen testing. One patient had a high baseline hydrogen excretion (25 ppm) with no subsequent rise, while the second patient had an acceptable baseline level $(6 \mathrm{ppm})$ and no further elevation from his baseline level.

\section{DISCUSSION}

Children with toddler's diarrhea are likely to be managed most often at the primary care level. These patients have good oral intake, normal growth and no concerning signs for infection or inflammation. This condition remains poorly understood and may encompass a heterogeneous group of disease entities. The differential diagnosis includes a variety of disorders including carbohydrate malabsorption. Congenital sucrase-isomaltase deficiency classically presents with significant diarrhea in early childhood with introduction of sucrose into the diet. Several studies have documented mild or delayed symptoms, most likely related to the amount sucrose consumption $[13,14]$ which may lead to misdiagnosis as nonspecific diarrhea. Additional factors may contribute to symptom severity including gastric emptying rate, activity and concentration of colonic bacteria and colonic absorptive capacity.

In our cohort, three patients responded to standard dietary manipulation with restriction of juice and excessive fluid intake and increases in fiber consumption. Some studies have demonstrated benefit from increasing fat intake [6, 7]. Diet adjustment remains the simplest solution and should be re-explained and emphasized to families even those who report previous failures. Limited screening tests are often performed and should serve to reassure the physician and parents that no serious underlying disorder exits. In patients with other symptoms such as anorexia, failure to thrive, bloody stools, abdominal distension or pain, further evaluation is needed.

Several tests exit to make the diagnosis of CSID. Invasive testing with determination of intestinal disaccharidase levels is the gold standard. Several important factors affect the accuracy of such testing including biopsy location within the small intestine, number of biopsies obtained and tissue handling after biopsy. Upper endoscopy with small bowel biopsies was not performed in our patients as it was hard to ethically and economically justify in patients who had normal appetite, growth and development. Sucrose breath hydrogen testing has been validated in children with sucrose malabsorption [21]. This was offered but only accepted by 
two families. False negatives occur in non-hydrogen producers in less than $5 \%$ of children in the absence of other causes such as recent antibiotic use [22]. Much higher rates of nonhydrogen producers (up to $20 \%$ ) have been reported in adult studies [23]. High baseline hydrogen breath levels, as noted by one of our patients, can make this test less useful and may suggest small bowel bacterial overgrowth. We opted to use a short sacrosidase therapeutic trial to assess for clinical response. The limitations of this approach include difficulty in differentiating sucrase deficiency (primary or secondary) from high sucrose consumption (although this was supposedly minimized or eliminated in our cohort). Confirmatory testing, preferably with small bowel biopsies, should still be performed prior to committing patients to life-long sucrose free diet or sacrosidase supplementation.

In our cohort of children with toddler's diarrhea, sacrosidase supplementation demonstrated a clinical benefit in a subset of patients by decreasing stool frequency and/or improving stool consistency. The cohort size was small and long term follow-up was not available in patients who responded. The observed response may be due to excessive sucrose in the children's diet which may still be underappreciated by the family, or it may be due unrecognized complete or partial sucrase deficiency. Clinical nonresponders may still have had CSID with low isomaltase activity, but remained symptomatic despite sacrosidase supplementation because of starch consumption.

A short therapeutic sacrosidase trial in patients with toddler's diarrhea may be attempted but the limitations of such intervention should be clarified to the families of such patients. A larger scale study with more invasive confirmatory testing is needed to assess the long term benefit of such an intervention.

\section{ACKNOWLEDGEMENTS}

We greatly appreciate the support from Dr. Tammy Meehan, Dr. Dawn Ebach, Sally Arensdorf, RN and Judith Heckman, PA-C. This research was partially supported by an educational Grant from QOL Medical, Kirkland, WA, USA.

\section{REFERENCES}

[1] Davidson M, Waserman R. The irritable colon of childhood (chronic nonspecific diarrhea syndrome). J Pediatr 1966; 69: 102738 .

[2] Huffman S. Toddler's diarrhea. J Pediatr Health Care 1999; 13(1): 32-3.

[3] Kneepkens CM, Hoekstra JH. Chronic nonspecific diarrhea of childhood: pathophysiology and management. Pediatr Clin North Am 1996; 43: 375-90.
[4] Hamdi I, Dodge JA. Toddler diarrhoea: observations on the effects of aspirin and loperamide. J Pediatr Gastroenterol Nutr 1985; 4: 362-65.

[5] Judd RH. Chronic nonspecific diarrhea. Pediatr Rev 1996; 17(11): 379-84.

[6] Treem WR. Chronic nonspecific diarrhea of childhood. Clin Pediatr (Phila) 1992; 31(7): 413-20.

[7] Boyne LJ, Kerzner B, McClung HJ. Chronic nonspecific diarrhea: the value of a preliminary observation period to assess diet therapy. Pediatrics 1985; 76(4): 557-61.

[8] Gray GM, Lally BC, Conklin KA. Action of intestinal sucraseisomaltase and its free monomers on an alpha-limit dextrin. J Biol Chem 1979; 254: 6038-43.

[9] Skovbjerg H, Krasilnikoff PA. Maltase-glucoamylase and residual isomaltase in sucrose intolerant patients. J Pediatr Gastroenterol Nutr 1986; 5: 365-371.

[10] Treem WR, Ahsan N, Sullivan B, et al. Evaluation of liquid yeastderived sucrase enzyme replacement in patients with sucraseisomaltase deficiency. Gastroenterology 1993; 105: 1061-68.

[11] Kelly JJ, Alpers DH. Properties of human intestinal glucoamylase. Biochim Biophys Acta 1973; 315: 113-22.

[12] Treem WR. Clinical heterogeneity in congenital sucrase-isomaltase deficiency. J Pediatr 1996; 128: 727-29.

[13] Treem WR. Congenital sucrase-isomaltase deficiency. J Pediatr Gastroenterol Nutr 1995; 21: 1-14

[14] Baudon JJ, Veinberg F, Thioulouse E, Morgant G, Aymard P, Charritat JL. Sucrase-Isomaltase Deficiency: Changing Pattern over Two Decades. J Pediatr Gastroenterol Nutr 1996; 22(3): 28488.

[15] Sonntag WM, Brill ML, Troyer WG, Jr., Welsh JD, Semenza G, Prader A. Sucrose-isomaltose malabsorption in an adult woman. Gastroenterology 1964; 47: 18-25.

[16] Ringrose RE, Preiser H, Welsh JD. Sucrase-isomaltase (palatinase) deficiency diagnosed during adulthood. Dig Dis Sci 1980; 25: 384 87.

[17] Muldoon C, Maguire P, Gleeson F. Onset of sucrase-isomaltase deficiency in late adulthood. Am J Gastroenterol 1999; 94: 229899.

[18] Gudmand-Høyer E. Sucrose malabsorption in children: a report of thirty-one Greenlanders. J Pediatr Gastroenterol Nutr 1985; 4(6): 873-7.

[19] Antonowicz I, Lloyd-Still MB, Khaw KT, Shwachman H. Congenital sucrase-isomaltase deficiency. Observations over a period of 6 years. Pediatrics 1972; 49: 847-53.

[20] Treem WR, McAdams L, Stanford L, Kastoff G, Justinich C, Hyams J. Sacrosidase therapy for congenital sucrase-isomaltase deficiency. J Pediatr Gastroenterol Nutr 1999; 28: 137-42.

[21] Perman JA, Barr RG, Watkins JB. Sucrose malabsorption in children: noninvasive diagnosis by interval breath hydrogen determination. J Pediatr 1978; 93: 17-22.

[22] Davidson GP, Robb TA. Value of breath hydrogen analysis in management of diarrheal illness in childhood: comparison with duodenal biopsy. J Pediatr Gastroenterol Nutr 1985; 4(3): 381-7.

[23] Gilat T, Ben Hur H, Gelman-Malachi E, Terdiman R, Peled Y. Alterations of the colonic flora and their effect on the hydrogen breath test. Gut 1978; 19: 602-5. 